Editorial

\title{
Introduction to the special section on Image and Video Processing
}

Image and video processing research has undergone enormous changes and development in recent decades. This is due to the rapid advancements of visual technologies and their use in portable devices, medical imaging, and video streaming, for applications in photography, social media, entertainment, security and health care, among others. As images and video have become main means of communication and verification, the creation, transfer, and storage of high-quality images and videos require special attention. The necessity for retrieving relevant information from images and video, and the technological advancements have led fundamental research looking for novel processing techniques in image enhancement for reconstruction, restoration, de-noising, improvement of resolution and reduction of artifacts and noise; image segmentation for extracting an object from a background; face recognition for identification and classification; pattern recognition for monitoring and diagnosis; security and compression for improving the protection of information, optimizing its storage, and maintaining its quality; image classification for object recognition, description, and matching of feature points; image annotation for interpretation of contents through words, keywords or comments; and image modeling to detect depth information of a target object. In particular, the increasing usage of Internet, social networks and wireless communication technologies with high quality image streaming have enormous needs for image and video processing. These needs have proven the importance of performing research in this area for continuous innovation.

\section{Quick facts about this special issue}

This is the eighth special section on image and video processing; the previous seven were published in August 2016, August 2015, November 2014, April 2014, April 2013, September 2012, and September 2011. We foresee more special sections on image and video processing as the area is open to new developments with many innovative and highly productive researchers. This issue focuses on recent efforts and results for addressing image and video processing challenges, and the novel and original answers to these demands. It is intended to present a big picture of image and video processing by introducing recent advances and research in the field. A total of 69 submitted papers were considered. Each paper was peer reviewed by three or more renowned experts during the assessment process. After evaluating the overall scores, 38 highquality articles were chosen by the guest editors and approved by the Editor-in-Chief for inclusion in this special section. The selected articles are diverse in terms of techniques and applications. They present in-depth studies of practical issues and challenging problems in image and video processing; we hope they will be valuable to researchers and practitioners in this field.

\section{Scanning the issue}

The papers in this special issue can be grouped in several main areas: 1) Pattern Recognition and Image Segmentation, 2) Image Enhancement, 3) Image Security and Compression, 4) Image Classification, 5) Image Annotation, and 6) Image Modeling. Pattern Recognition and Image Segmentation are critical areas of research in image processing. Pattern Recognition, as its name suggests, allows the identification of patterns and regularities in data; on the other hand, Image Segmentation allows locating objects and boundaries in images by grouping pixels that share certain characteristics. This special issue has twelve manuscripts in these areas. The first one proposes a face verification framework using 2D and 3D, introducing a face descriptor based on local statistics of 2D and 3D images. The second one proposes a 3D face recognition method using the local covariance descriptor and Riemannian kernel sparse coding to evaluate the intrinsic correlation of extracted 
features and improve the 3D face recognition. In the third manuscript, a hand-crafted local quadruple pattern is proposed for facial image recognition and retrieval by encoding relations amongst the neighbors in quadruple space. The fourth article describes a parametric active contour model based on the texture describing properties derived from coefficients of the two-dimensional Discrete Cosine Transform for segmenting texture objects against complex backgrounds. In the fifth one, a hybrid method for image segmentation containing single and multiple objects is proposed, using a two-dimensional Gabor filter to enhance boundaries for extracting transition regions. The sixth paper presents a fully-automated microarray gridding algorithm for block finding in an image, image contrast enhancement, and identification of image objects including spots and artifacts. The seventh one presents a medical image segmentation technique to improve accuracy, complexity, and robustness preserving the advantages of existing methods, achieving high image segmentation accuracy. In the eighth one, an iris localization algorithm for visible wavelength eye images is proposed by suppressing light reflections and sharpening intensity variations in the Red component of input color eye image. The ninth one proposes the concept of class-specific dictionaries for iris recognition and a modular approach to diminish noise due to imperfect segmentation of iris regions using a partitioning and fusion of individual decisions for all sectors in an iris-image. The tenth article develops an automated computer aided diagnosis system for new abnormal blood vessel detection on retinal images by thick and thin vessels extraction from the entire vessel map. The eleventh one presents an active contour model based on median absolute deviation for remote sensing river image segmentation using the media absolute deviation for defining the external constraint terms, and fusing within-cluster variances and median absolute deviations to accelerate the model evolution. In the twelfth article, dust particles in images are identified using a set of salient visual descriptors that comprise localized color, texture and shape saliency features, which are further incorporated into a machine learning approach for performing the particle identification.

There are eleven articles in the area of Image Enhancement for applications such as image reconstruction and restoration, identification and assessment, depth of field, reduction of artifacts, denoising, and quality improvement. Image Enhancement plays a significant role in modern applications such as auto-focusing, remote sensing of the environment, object tracking, restoration, noise removal, object sharpening, quality assessment and improvement. When an image is captured there is some information loss due to noise like fog, cloudy sky, atmospheric reflection, acquisition errors, and so on. This information loss decreases the resolution and contrast of the image. Image Enhancement improves the image contrast so that it is pleasant to a human viewer and useful to apply computer based operations. In the first article, a regulation based super resolution reconstruction method of images is proposed by imposing two regularization constraints in order to maintain consistency of structural features, and preserve the complete set of high frequency components. The second one investigates the potential reduction in execution time of super-resolution methods for satellite images by selecting tasks that can be parallelized using general purpose computing on graphics processing units and compute unified device architecture (CUDA). In the third one, an interpolation-based single-frame image super-resolution approach using orthogonal rotation invariant moments is proposed for noise-free and noisy low-resolution images corrupted with uniform blur and Gaussian noise. The fourth article proposes learning based approaches for single image super-resolution using sparse representation and neighbor embedding to recover high-resolution image patches from the low resolution ones. In the fifth article, the maximum likelihood expectation maximization algorithm and ultrasonic time of flight data are used for obtaining a high quality image reconstruction to evaluate concrete filled steel tubes by identifying their position and shape. The sixth one presents a pixelbased multi-focus image fusion method that generates an all-in-focus image by combining images that are acquired from the same point of view with different focus settings. The seventh article introduces a deblurring model to explore the image sparsity and preserve the structure information of different scales by considering non-local constraint terms to exploit the self-similarity of non-local image patches. In the eight one, the nonlocal means method is extended using Zernike moments for image sequence denoising, reducing significantly the number of computations needed for block matching and weight computation. In the ninth article, a theoretical perspective is adopted for image dehazing, which considers the degraded image as a product contaminated by noise. Two maps are constructed to label the noise severity and atmospheric light. The parameters involved are optimized via Particle Swarm Optimization with a penalty function in terms of hue change. The tenth article proposes a computationally less expensive approach for contrast enhancement that overcomes the mean-shift problem by suing quantile-based division of the histogram equalization of a given image. In the eleventh article, a fusion algorithm to improve the quality of infrared and visible light images is introduced by combining object region detection with the non-subsampled contourlet transform.

With the rapid development of Internet and the emerging communication technologies; information security, intellectual property protection, and image compression are of great concern. This has led to extensive study of data encryption, digital signature, authentication, watermarking methods, and encoding. There are seven papers regarding Image Security and Compression in this special issue. In the first one, logistic map is employed for encrypting gray-scale images by dividing them into blocks and encrypting them using XOR operation and chaotic windows. The second article presents a multipleimage encryption algorithm based on mixed image elements and piecewise linear chaotic maps. The third one presents an algorithm for image quantum encryption introducing the physical basis of key generation and distribution for image quantum encryption. In the forth article, proposes a geometrically invariant watermarking method based on moments of polar harmonic transforms, which are computed using a simplified trigonometric formula, and supports geometric attacks. In the fifth one, a forgery detection method that depends on the discrete wavelet transform as well as the discrete cosine transform for feature reduction is presented and tested through a mask-based tampering method. The sixth article proposes a scheme for detecting multiple types of image forgeries through spatial rich model combined with local binary pattern. In the 
seventh article, an adaptive synthetic aperture radar image compression method is presented that consists of three stages: training stage, coding stage and decoding stage for constructing wavelet dictionaries, transforming the image into different frequency sub-bands, and reconstructing the image, respectively.

A fundamental problem in computer vision is Image Classification, which implicates the detection, description, and matching of feature points, which forms the basis of many applications such as image registration, object recognition, and image retrieval, among many others. In this special issue, six manuscripts that consider image classification through different approaches are included. In the first paper, a method for recognizing dynamic texture using local binary pattern is proposed, which differentiates among distinct structural patterns using information from a local patch combined with shape information. The second one introduces a parallel crossing deep convolutional neural network to simulate the human visual system for image classification through the concepts of super convolutional kernel and sub convolutional kernel in accordance with the process that human observes objects. The third manuscript presents a comparison of feature points on multisensor images, which possess non linear intensity changes, to identify robust feature points for image to image matching tasks on multisensor images. In the fourth paper, a mean-based adaptive random sample consensus method is proposed for extracting and matching features in images by using the distance variance in addition to the mean value. The fifth one, a brain-inspired computing model for target classification and recognition is proposed using cognitive computing and deep learning for extracting hierarchical features from remote-sensing images. The sixth paper uses feature space discriminant analysis to extract features for spectral-spatial classification of hyperspectral images through spatial features with class discrimination and little redundant information.

Another increasingly important image processing area is Image Annotation, which is very useful in the fields of security, geographical systems, and medicine, among others. The advances in information technology have induced an explosive growth of image databases. Image annotation requires and demands effective and efficient tools to produce words, keywords or comments for an image or for a selected part of it. This special issue has one Image Annotation paper, which proposes a genetic-based prototyping for automatic image annotation that adopts a genetic based algorithm for retrieving neighbor images by obtaining an optimal prototype for a query image. Candidate tags are generated using methods such as voting.

Finally, 3D Image Modeling allows detecting depth information of objects making possible to represent them with better quality and as close as possible to their actual size; hence, 3D image modeling is quite useful on applications like medicine education, manufacturing, and machinery, among many others. This special issue includes one manuscript on 3D image modeling, which introduces a norm-entropy-based method that uses a modified neutrosophic set segmentation to improve the quality of a single object 3D modeling.

The guest editors would like to thank the authors for their contributions to the special issue and all the reviewers for their constructive comments. We are also grateful to the Editor-in-Chief, Manu Malek, for his support and encouragements and the Journal's editorial staff for their help in the preparation of this special issue.

Guest Editors

Eduardo Cabal-Yepez

Division de Ingenierias, Campus Irapuato-Salamanca, Universidad de Guanajuato, Mexico

Guillermo Botella

Department of Computer Architecture and Automation, Complutense University of Madrid, Spain

Amir Hooshang Mazinan

Electrical Engineering, Islamic Azad University, Iran

Joao P. Papa

Department of Computing, School of Sciences, Sao Paulo State University

E-mail addresses: e.cabalyepez@gmail.com, educabal@ugto.mx (E. Cabal-Yepez), gbotella@ucm.es (G. Botella), ahmazinan@gmail.com, mazinan@azad.ac.ir (A.H. Mazinan), papa@fc.unesp.br (J.P. Papa) 

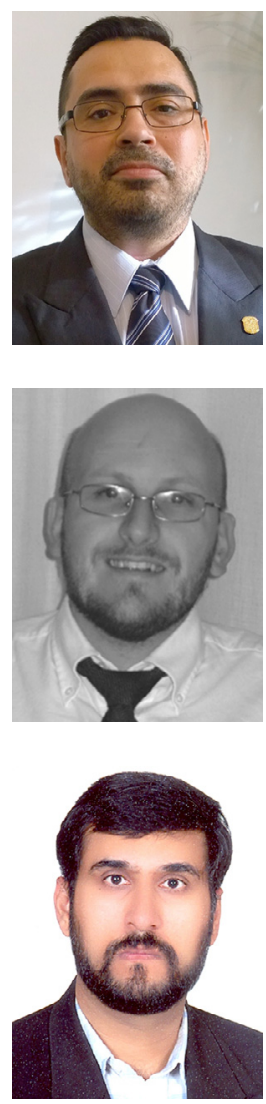

Amir Hooshang MAZINAN received the Ph.D. degree in 2009 in Control Engineering. Dr. Mazinan is the Associate Professor and also the Director of Control Engineering Department at Islamic Azad University, South Tehran Branch, Tehran, Iran, since 2009. $\mathrm{He}$ is now acting as the Associate Editor in Transactions of the Institute of Measurement and Control (Sage publisher) and the Guest Editor in Computers \& Electrical Engineering Journal (Elsevier Publisher), as well. Moreover, he is a member of Editorial Board in three international journals and also a member of programming committee in four international conferences. He has more than 100 journal and conference papers in so many reputable publishers. His current research interests include intelligent systems, model based predictive control, over-actuated space systems modeling and control, time-frequency representation, filter banks, wavelet theory and image-video processing.

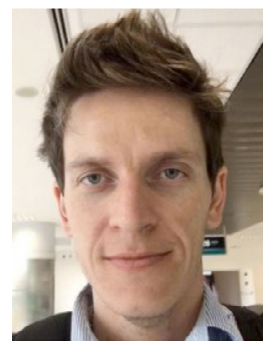

João P. Papa received his B.Sc. in Information Systems from the São Paulo State University, SP, Brazil. In 2005, he received his M.Sc. in Computer Science from the Federal University of São Carlos, SP, Brazil. In 2008, he received his Ph.D. in Computer Science from the University of Campinas, SP, Brazil. During 2008-2009, he had worked as a post-doctorate researcher at the same institute, and during 2014-2015 he worked as a visiting professor at Harvard University. He has been Professor at the Computer Science Department, São Paulo State University, since 2009, and his research interests include machine learning, pattern recognition, and image processing. Currently, he serves as an Associate Editor for IEEE Signal Processing Letters, as well as he is a member of the editorial board of the International Journal of Bio-Inspired Computation and the Computer Methods in Biomechanics and Biomedical Engineering: Imaging \& Visualization journal. 